\title{
A UNIFIED VIEW OF LIBOR MODELS
}

\author{
KATHRIN GLAU, ZORANA GRBAC, AND ANTONIS PAPAPANTOLEON
}

\begin{abstract}
We provide a unified framework for modeling LIBOR rates using general semimartingales as driving processes and generic functional forms to describe the evolution of the dynamics. We derive sufficient conditions for the model to be arbitrage-free which are easily verifiable, and for the LIBOR rates to be true martingales under the respective forward measures. We discuss when the conditions are also necessary and comment on further desirable properties such as those leading to analytical tractability and positivity of rates. This framework allows to consider several popular models in the literature, such as LIBOR market models driven by Brownian motion or jump processes, the Lévy forward price model as well as the affine LIBOR model, under one umbrella. Moreover, we derive structural results about LIBOR models and show, in particular, that only models where the forward price is an exponentially affine function of the driving process preserve their structure under different forward measures.
\end{abstract}

\section{INTRODUCTION}

The LIBOR and EURIBOR interest rates rank among the most important interest rates worldwide. They are determined on a daily basis by a panel of banks for a number of maturities, while LIBOR is also determined for several currencies. LIBOR and EURIBOR serve as underlying rates for an enormous amount of financial transactions. In 2012, the outstanding values of contracts with LIBOR as reference were estimated at roughly summing up to 300 trillion USD; see Wheatley (2012). Therefore, the development of suitable policies and regulations for the fair calculation of LIBOR and EURIBOR, as well as of mathematical models for the fair evaluation of interest rate products, is essential for the financial industry and also serves the general public interest.

The modeling of the dynamics of LIBOR and EURIBOR rates is a challenging task due to the high dimensionality of the modeled objects. The major difference, from a modeling point of view, between interest rates and stock prices lies in the fact that stock prices are observed at each time point as a single value, once the difference between bid and ask prices is ignored, while interest rates are observed at each time point for several maturities. Moreover, these different rates (for the different maturities) are interdependent. Their joint modeling is indispensable because they jointly enter already the basic interest rate derivatives as underlying rates. In addition, the rates for different period lengths can no longer be derived from simple no-arbitrage relations.

2010 Mathematics Subject Classification. 91G30, 60G44.

Key words and phrases. LIBOR, forward price, semimartingales, LIBOR market models, Lévy forward price models, affine LIBOR models.

Financial support from the PROCOPE project "Financial markets in transition: mathematical models and challenges" and the Europlace Institute of Finance project "Post-crisis models for interest rate markets" is gratefully acknowledged. 
Indeed, the financial crisis of 2007-2009 has fundamentally changed the attitude of market participants towards risks in the interbank sector, regarding in particular counterparty and liquidity risk, which have a direct effect on the LIBOR rates for different lending periods; see, for example, Filipović and Trolle (2013). Summarizing, LIBOR modeling presents a challenge to jointly model the rates for different maturities and periods in an arbitrage-free way and such that the resulting pricing formulas are fast and accurately computable for all liquid derivatives, such as caps and swaptions. In this work, we provide a unified mathematical foundation for some of the most important of the existing LIBOR models in the literature. On this basis we gain valuable structural insight in modeling LIBOR rates. In particular we derive sufficient conditions for the validity of mandatory model features such as arbitrage-freeness and investigate those that typically support computational ease.

The seminalarticles by Brace, Gạtarek, and Musiela (1997) and Miltersen, Sandmann, and Sondermann (1997) introduced the LIBOR Market Model (LMM), that became known also as the BGM model. The celebrity of the model certainly is, at least partly, owed to the fact that the BGM model reproduces the market standard Black's formula for caps. Moreover, the backward construction of LIBOR rates in the LMM presented in Musiela and Rutkowski (1997) has proven to be extendible beyond models driven by Brownian motion. The article by Eberlein and Özkan (2005) introduced one of the first LIBOR models driven by jump processes and also proposed the Lévy-driven forward price model. In Jamshidian $(1997 ; 1999)$ LIBOR models driven by general semimartingales were presented. In interest rate modeling, jump processes have several advantages. Firstly, just as in stock price modeling, their distributional flexibility allows to better capture the empirical distributions of logarithmic returns, see for instance Eberlein and Kluge (2006a; 2006b). Secondly, the traditional way to jointly model the rates for different maturities would suggest to introduce one component of a multi-dimensional Brownian motion for each maturity, so as to introduce one stochastic factor for each source of risk. A Lévy process with an infinite jump activity, in contrast, introduces infinitely many sources of risk, already as a one-dimensional process. In this regard, jump processes show their potential to reduce the dimension of the related computational problems for pricing and hedging. However, they also bring along a new level of technical challenges, in particular the measure changes between forward measures become more involved and the backward construction typically requires a more sophisticated justification. Additionally, various extensions of the LIBOR market model to stochastic volatility have also appeared in the literature, cf. Wu and Zhang (2006), Belomestny, Mathew, and Schoenmakers (2009) and Ladkau, Schoenmakers, and Zhang (2013). Recently, a modeling approach under one single forward measure, the terminal measure, has been proposed in Keller-Ressel, Papapantoleon, and Teichmann (2013), which is based on affine processes. We refer to Schoenmakers (2005) and Papapantoleon (2010) for an overview of the modeling approaches and the existing literature. Regarding the post-crisis LIBOR models we refer to Bianchetti and Morini (2013) and Grbac and Runggaldier (2015).

In view of the high level of technical sophistication that LIBOR models have reached in today's literature and also of the new demands they are faced with, we propose an abstract perspective on LIBOR modeling in order to obtain: 
- a unified view on different modeling approaches, such as the LIBOR market models, the Lévy forward price models and the affine LIBOR models;

- transparent conditions that guarantee:

$\circ$ positivity of bond prices and arbitrage-freeness - the fundamental model requirements;

- martingality of the forward prices under their corresponding forward measures, which paves the way for change of numeraire techniques and tractable pricing formulas;

o structure preservation under different forward measures, a feature that is beneficial in connection with change of numeraire techniques;

- the validity of further desirable model properties that lead to analytically tractable models.

This article is structured as follows: in Section 2 we introduce the main modeling objects and formalize model axioms as well as desirable model properties that entail computational tractability. In Section 3, we provide two general modeling approaches based on general semimartingales and generic functional forms for the evolution of rates, and derive sufficient conditions for the arbitrage-freeness of the models and for the forward price processes to be uniformly integrable martingales under their corresponding forward measures; positivity of bond prices holds by construction. On this basis we derive conditions that imply positivity of LIBOR rates and ensure computational tractability. As an interesting additional insight we show that essentially only models in which the forward price processes are exponentials of an affine function of a semimartingale are structure preserving under different forward measures. In Section 4, we present several LIBOR models in the guise of the general modeling framework and investigate sufficient conditions that lead to further essential model features. Finally, required results from semimartingale theory are derived in the appendix.

\section{Axioms AND DESIRABle PROPERTIES}

Let $\left(\Omega, \mathcal{F}=\mathcal{F}_{T_{*}}, \mathbb{F}=\left(\mathcal{F}_{t}\right)_{t \in\left[0, T_{*}\right]}, \mathbb{P}_{N}\right)$ denote a complete stochastic basis in the sense of Jacod and Shiryaev (2003, Def. I.1.3), where $T_{*}$ denotes a finite time horizon. Consider a discrete tenor structure $\mathcal{T}:=\left\{0=T_{0}<\ldots<T_{N} \leq\right.$ $\left.T_{*}\right\}$ with $\delta_{k}=T_{k}-T_{k-1}$ for $k \in \mathcal{K}:=\{1, \ldots, N\}$, and define $\overline{\mathcal{K}}:=\mathcal{K} \backslash\{N\}$. We assume that zero-coupon bonds with maturities $T_{1}, \ldots, T_{N}$ are traded in the market and denote by $B\left(t, T_{k}\right)$ the time- $t$ price of the zero-coupon bond with maturity $T_{k}$, for all $k \in \mathcal{K}$. We associate to each date $T_{k}$ the numeraire pair $\left(B\left(\cdot, T_{k}\right), \mathbb{P}_{k}\right)$, meaning that bond prices discounted by the numeraire $B\left(\cdot, T_{k}\right)$ are $\mathbb{P}_{k}$-local martingales, for all $k \in \mathcal{K}$. The measures $\mathbb{P}_{k}$ are then called forward (martingale) measures. Moreover, let $\mathcal{M}_{\mathrm{loc}}(\mathbb{P})$ denote the set of local martingales with respect to the measure $\mathbb{P}$.

The forward $L I B O R$ rate, denoted by $L\left(t, T_{k}\right)$, is a discretely compounded interest rate determined at time $t$ for the future accrual interval $\left[T_{k}, T_{k+1}\right]$. It is related to bond prices via

$$
L\left(t, T_{k}\right)=\frac{1}{\delta_{k}}\left(\frac{B\left(t, T_{k}\right)}{B\left(t, T_{k+1}\right)}-1\right), \quad t \in\left[0, T_{k}\right]
$$


for $k \in \overline{\mathcal{K}}$. The forward price process $F\left(\cdot, T_{k}, T_{n}\right)$ is defined as follows

$$
F\left(t, T_{k}, T_{n}\right)=\frac{B\left(t, T_{k}\right)}{B\left(t, T_{n}\right)}, \quad t \in\left[0, T_{k} \wedge T_{n}\right],
$$

for all $k, n \in \mathcal{K}$. The forward LIBOR rate $L\left(t, T_{k}\right)$ and the forward price $F\left(t, T_{k}, T_{k+1}\right)$ are connected via

$$
F\left(t, T_{k}, T_{k+1}\right)=1+\delta_{k} L\left(t, T_{k}\right) .
$$

We will describe in the sequel several axioms and properties that LIBOR models should posess in order to be economically meaningful on the one hand, and applicable in practice on the other. In particular, we will distinguish between three different groups of attributes. The first group consists of necessary axioms, which are needed to build a sound financial model. These are:

(A1) Bond prices are positive, i.e. $B\left(\cdot, T_{k}\right)>0$ for all $k \in \mathcal{K}$;

$(\mathbb{A} 2)$ The model is arbitrage-free, i.e. $\frac{B\left(\cdot, T_{k}\right)}{B\left(\cdot, T_{N}\right)} \in \mathcal{M}_{\text {loc }}\left(\mathbb{P}_{N}\right)$ for all $k \in \mathcal{K}$.

The first axiom is justified since bond prices are traded assets with a positive payoff, thus should have a positive price. The second axiom precludes the existence of arbitrage opportunities and could be equivalently formulated under any forward measure, i.e. the model is arbitrage-free if $\frac{B\left(\cdot, T_{k}\right)}{B\left(\cdot, T_{n}\right)} \in \mathcal{M}_{\mathrm{loc}}\left(\mathbb{P}_{n}\right)$ for all $k \in \mathcal{K}$ and some $n \in \mathcal{K}$; see also Musiela and Rutkowski $(2005, \S 14.1 .3)$ and Klein, Schmidt, and Teichmann (2015).

The second group consists of tractability properties, which simplify computations in the model. Out of several possible choices, we will concentrate on the following:

$(\mathbb{B} 1)$ Forward prices are true martingales, i.e. $\frac{B\left(\cdot, T_{k}\right)}{B\left(\cdot, T_{N}\right)} \in \mathcal{M}\left(\mathbb{P}_{N}\right)$ for all $k \in \mathcal{K}$.

$(\mathbb{B} 2)$ The model is structure preserving, i.e. the semimartingale characteristics of the driving process are transformed in a deterministic way under forward measures.

$(\mathbb{B} 3)$ Each LIBOR rate is a Markov process under its corresponding forward measure.

$(\mathbb{B} 4)$ The initial LIBOR rates are direct model inputs.

These properties are not necessary to build an arbitrage-free model, but are very convenient in several aspects. The first property allows to compute option prices as conditional expectations and to relate the forward measures via a density process. Hence, several option pricing formulas can be simplified considerably by changing to a more convenient forward measure. The second property yields that the processes driving each LIBOR rate remain in the same class of processes under each forward measure. Moreover, (B1) combined with (BB2) typically allows to derive closed-form or semi-analytical pricing formulas for liquid products such as caps and swaptions. (B3) also allows to simplify certain option pricing problems and use PDE methods. Additionally, if the initial term structure is a direct input in the model, i.e. ( $\mathbb{B} 4$ ) holds, then we avoid using a numerical procedure to fit the currently observed bond prices.

Finally, we shall also discuss the following model property:

$(\mathbb{C})$ LIBOR rates are always non-negative. 
Until the recent financial crisis, LIBOR rates were always non-negative, hence the possibility of rates becoming negative has been considered as a drawback of a model. As a consequence, several LIBOR models have been designed to produce non-negative LIBOR rates. Nowadays, the quoted LIBOR rates are at extremely low levels and even negative LIBOR rates for several tenors have been reported over longer time periods, which prompts us to take this into account in the modeling. Therefore, it is important to know which models allow for negative rates as well as which of the existing models for positive rates can easily be adapted to allow the rates to go below zero. Moreover, the techniques used to construct non-negative LIBOR rates can often be adapted to model other related non-negative quantities such as spreads in multiple curve models.

\section{A Unified CONSTRUCTION OF LIBOR MODELS}

Models for the evolution of LIBOR rates are constructed in the literature either using a backward induction approach, where rates are specified successively under different forward measures, or by modeling all rates simultaneously under one measure, typically the terminal forward measure. The former approach has been used for the construction of LIBOR market models and forward price models, while the latter is used for affine LIBOR models and Markov functional models. The aim of this section is to offer a unified construction of LIBOR models by emphasizing the common features in both approaches.

3.1. Modeling rates via backward induction. The aim of this subsection is to formulate sufficient conditions and to present a generic construction of LIBOR (market) models using the backward induction approach. The driving process is a general semimartingale and the functional form of the dynamics is also generic.

The following key observations of Musiela and Rutkowski (1997) lie at the heart of the constructions via backward induction:

- A model for the LIBOR rates $\left(L\left(\cdot, T_{k}\right)\right)_{k \in \overline{\mathcal{K}}}$ is arbitrage-free if $L\left(\cdot, T_{k}\right)$ is a $\mathbb{P}_{k+1}$-local martingale for all $k \in \overline{\mathcal{K}}$.

- The forward measures $\left(\mathbb{P}_{k}\right)_{k \in \overline{\mathcal{K}}}$ are related via the Radon-Nikodym derivatives

$$
\frac{\mathrm{dP}}{\mathrm{dP}_{k+1}}=\frac{1+\delta_{k} L\left(T_{k}, T_{k}\right)}{1+\delta_{k} L\left(0, T_{k}\right)}, \quad \text { for all } k \in \overline{\mathcal{K}}
$$

Therefore, in order to construct a LIBOR model it suffices to specify the dynamics either of the LIBOR rate $L\left(\cdot, T_{k}\right)$ itself or of the forward price process $F\left(\cdot, T_{k}, T_{k+1}\right)=1+\delta L\left(\cdot, T_{k}\right)$ for all $k \in \overline{\mathcal{K}}$, and both choices determine the densities in (3.1) as well.

Our construction is based on specifying an exponential semimartingale for the dynamics of the forward price process with the following functional form:

$$
F\left(\cdot, T_{k}, T_{k+1}\right)=\mathrm{e}^{f^{k}(\cdot, X)},
$$

where $f^{k}$ are functions for each $k \in \overline{\mathcal{K}}$ and $X$ is a semimartingale. This approach unifies the construction of the LIBOR market models and the forward price models by appropriate choices of $f^{k}$ and $X$ that will be discussed in Section 3 . 
Consider an $\mathbb{R}^{d}$-valued semimartingale $X=\left(X_{t}\right)_{0 \leq t \leq T_{N}}$ on $\left(\Omega, \mathcal{F}, \mathbb{F}, \mathbb{P}_{N}\right)$ and a collection of functions $f^{k}:\left[0, T_{N}\right] \times \mathbb{R}^{d} \rightarrow \mathbb{R}$ for all $k \in \mathcal{K}$, which satisfy the following assumptions:

$(\mathbb{L} \mathbb{I P})$ The function $f^{k}$ belongs to $C^{1,2}\left(\left[0, T_{N}\right] \times \mathbb{R}^{d}\right)$ and is globally Lipschitz, i.e.

$$
\left|f^{k}(t, x)-f^{k}(t, y)\right| \leq K^{k}|x-y|,
$$

for every $t \in\left[0, T_{N}\right]$ and any $x, y \in \mathbb{R}^{d}$, where $K^{k}>0$ is a constant.

$(\mathbb{I N T})$ The process $X$ is an $\mathbb{R}^{d}$-valued semimartingale with absolutely continuous characteristics $\left(b^{N}, c^{N}, F^{N}\right)$ under $\mathbb{P}_{N}$, such that the following conditions hold

$$
\int_{0}^{T_{N}} \int_{\mathbb{R}^{d}}\left\{|x|^{2} 1_{\{|x| \leq 1\}}+|x| \mathrm{e}^{K|x|} 1_{\{|x|>1\}}\right\} F_{t}^{N}(\mathrm{~d} x) \mathrm{d} t<C_{1}
$$

and

$$
\int_{0}^{T_{N}}\left\|c_{t}^{N}\right\| \mathrm{d} t<C_{2}
$$

for some constants $C_{1}, C_{2}>0$ and $K=\sum_{k=1}^{N-1} K^{k}$.

We denote by $\|\cdot\|$ the Euclidean norm on $\mathbb{R}^{d}$ and by $\langle\cdot, \cdot\rangle$ the associated scalar product.

Remark 3.1. The characteristic triplet of the semimartingale $X$ under the forward measure $\mathbb{P}_{k}$ is denoted by $\left(b^{k}, c^{k}, F^{k}\right)$, while the truncation function can always be chosen the identity (i.e. $h(x)=x$ ) due to (3.3). Moreover, we use the standard conventions $\sum_{\emptyset}=0$ and $\prod_{\emptyset}=1$.

Theorem 3.2. Consider an $\mathbb{R}^{d}$-valued semimartingale $X$ and functions $f^{k}$ such that Assumptions $(\mathbb{L} \mathbb{I P})$ and $(\mathbb{I N T})$ are satisfied for each $k \in \overline{\mathcal{K}}$. Assume that the forward price processes are modeled via

$$
F\left(t, T_{k}, T_{k+1}\right)=\mathrm{e}^{f^{k}\left(t, X_{t}\right)}, \quad t \in\left[0, T_{k}\right],
$$

and the following drift condition is satisfied

$$
\begin{aligned}
\left\langle\mathrm{D} f^{k}\left(t, X_{t-}\right), b^{N}\right\rangle= & -\frac{\mathrm{d}}{\mathrm{d} t} f^{k}\left(t, X_{t-}\right)-\frac{1}{2} \sum_{i, j=1}^{d} \mathrm{D}_{i j}^{2} f^{k}\left(t, X_{t-}\right)\left(c_{t}^{N}\right)^{i j} \\
& -\frac{1}{2}\left\langle\mathrm{D} f^{k}\left(t, X_{t-}\right), c_{t}^{N} \mathrm{D} f^{k}\left(t, X_{t-}\right)\right\rangle \\
& -\sum_{j=k+1}^{N-1}\left\langle\mathrm{D} f^{k}\left(t, X_{t-}\right), c_{t}^{N} \mathrm{D} f^{j}\left(t, X_{t-}\right)\right\rangle \\
- & \int_{\mathbb{R}^{d}}\left\{\left(\mathrm{e}^{f^{k}\left(t, X_{t-}+x\right)-f^{k}\left(t, X_{t-}\right)}-1\right)\right. \\
& \left.\times \prod_{j=k+1}^{N-1} \mathrm{e}^{f^{j}\left(t, X_{t-}+x\right)-f^{j}\left(t, X_{t-}\right)}-\left\langle\mathrm{D} f^{k}\left(t, X_{t-}\right), x\right\rangle\right\} F_{t}^{N}(\mathrm{~d} x)
\end{aligned}
$$


for each $k \in \overline{\mathcal{K}}$. Then, the measures $\left(\mathbb{P}_{k}\right)_{k \in \overline{\mathcal{K}}}$ defined via

$$
\frac{\mathrm{d} \mathbb{P}_{k}}{\mathrm{~d} \mathbb{P}_{k+1}}=\frac{\mathrm{e}^{f^{k}\left(T_{k}, X_{T_{k}}\right)}}{\mathrm{e}^{f^{k}\left(0, X_{0}\right)}}
$$

are equivalent forward measures and the forward prices processes $F\left(\cdot, T_{k}, T_{k+1}\right)$ are uniformly integrable martingales with respect to $\mathbb{P}_{k+1}$, for each $k \in \overline{\mathcal{K}}$. In particular, the model $\left(F\left(\cdot, T_{k}, T_{k+1}\right)\right)_{k \in \overline{\mathcal{K}}}$ is arbitrage-free and satisfies Axioms $(\mathbb{A} 1)$ and $(\mathbb{A} 2)$, as well as Property $(\mathbb{B} 1)$.

Proof. The statement is proved via backward induction, motivated by the backward construction of LIBOR and forward price models.

First step: We start from the forward price process $F\left(\cdot, T_{N-1}, T_{N}\right)$ whose dynamics are

$$
F\left(t, T_{N-1}, T_{N}\right)=\mathrm{e}^{f^{N-1}\left(t, X_{t}\right)}, \quad t \in\left[0, T_{N-1}\right],
$$

and examine its properties under the measure $\mathbb{P}_{N}$. The function $f^{N-1}$ satisfies $(\mathbb{L I P})$ and the process $X$ satisfies $(\mathbb{I N T})$, hence the process $f^{N-1}(\cdot, X)$ is an exponentially special semimartingale by Proposition A.2. Using Proposition A.1,

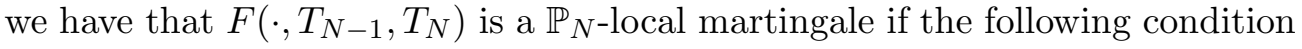
holds:

$$
\begin{aligned}
\left\langle\mathrm{D} f^{N-1}\left(t, X_{t-}\right), b^{N}\right\rangle \\
=-\frac{\mathrm{d}}{\mathrm{d} t} f^{N-1}\left(t, X_{t-}\right)-\frac{1}{2} \sum_{i, j=1}^{d} \mathrm{D}_{i j}^{2} f^{N-1}\left(t, X_{t-}\right)\left(c_{t}^{N}\right)^{i j} \\
\quad-\frac{1}{2}\left\langle\mathrm{D} f^{N-1}\left(t, X_{t-}\right), c^{N} \mathrm{D} f^{N-1}\left(t, X_{t-}\right)\right\rangle \\
\quad-\int_{\mathbb{R}^{d}}\left(\mathrm{e}^{f^{N-1}\left(t, X_{t-}+x\right)-f^{N-1}\left(t, X_{t-}\right)}-1-\left\langle\mathrm{D} f^{N-1}\left(t, X_{t-}\right), x\right\rangle\right) F_{t}^{N}(\mathrm{~d} x),
\end{aligned}
$$

which is actually $(\mathbb{D R} \mathbb{R} \mathbb{T})$ for $k=N-1$. Moreover, Proposition A.2 yields

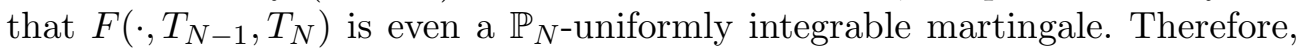
we can use $F\left(\cdot, T_{N-1}, T_{N}\right)$ as a density process to define the measure $\mathbb{P}_{N-1}$ via

$$
\left.\frac{\mathrm{d} \mathbb{P}_{N-1}}{\mathrm{dP}_{N}}\right|_{\mathcal{F} .}=\frac{F\left(\cdot, T_{N-1}, T_{N}\right)}{F\left(0, T_{N-1}, T_{N}\right)}=\frac{\mathrm{e}^{f^{N-1}(\cdot, X)}}{\mathrm{e}^{f^{N-1}\left(0, X_{0}\right)}},
$$

and the characteristics of the process $X$ under the measure $\mathbb{P}_{N-1}$ are provided by

$$
\begin{aligned}
b_{t}^{N-1}= & b_{t}^{N}+c_{t}^{N} \mathrm{D} f^{N-1}\left(t, X_{t-}\right) \\
& +\int_{\mathbb{R}^{d}}\left(\mathrm{e}^{f^{N-1}\left(t, X_{t-}+x\right)-f^{N-1}\left(t, X_{t-}\right)}-1\right) x F_{t}^{N}(\mathrm{~d} x) \\
c_{t}^{N-1}= & c_{t}^{N} \\
F_{t}^{N-1}(\mathrm{~d} x)= & \mathrm{e}^{f^{N-1}\left(t, X_{t-}+x\right)-f^{N-1}\left(t, X_{t-}\right)} F_{t}^{N}(\mathrm{~d} x) ;
\end{aligned}
$$

cf. Lemma A.4. 
Then, we proceed backwards by considering the 'next' forward price process $F\left(\cdot, T_{N-2}, T_{N-1}\right)$ with dynamics

$$
F\left(t, T_{N-2}, T_{N-1}\right)=\mathrm{e}^{f^{N-2}\left(t, X_{t}\right)}, \quad t \in\left[0, T_{N-2}\right],
$$

and verifying that subject to $(\mathbb{L} \mathbb{P}),(\mathbb{I N T})$ and $(\mathbb{D R} \mathbb{R} \mathbb{F})$ it is a $\mathbb{P}_{N-1}$-uniformly integrable martingale. Thus, it can be used as a density process to define the measure $\mathbb{P}_{N-2}$.

Next, we provide the general step of the backward induction.

General step: Let $k \in\{1, \ldots, N-1\}$ be fixed and consider the process $X$, the functions $f^{k+1}, \ldots, f^{N-1}$ and the measures $\mathbb{P}_{k+1}, \ldots, \mathbb{P}_{N}$ which are defined recursively via

$$
\left.\frac{\mathrm{d} \mathbb{P}_{k+1}}{\mathrm{~d} \mathbb{P}_{k+2}}\right|_{\mathcal{F} .}=\frac{F\left(\cdot, T_{k+1}, T_{k+2}\right)}{F\left(0, T_{k+1}, T_{k+2}\right)}=\frac{\mathrm{e}^{f^{k+1}(\cdot, X)}}{\mathrm{e}^{f^{k+1}\left(0, X_{0}\right)}} .
$$

Assume that the forward price processes $F\left(\cdot, T_{l}, T_{l+1}\right)$ have been modeled as exponential semimartingales according to (3.5) and are $\mathbb{P}_{l+1}$-uniformly integrable martingales, for all $l \in\{k+1, \ldots, N-1\}$. By repeatedly applying Lemma A.4, we derive the $\mathbb{P}_{k+1}$-characteristics of $X$, which have the form

$$
\begin{aligned}
b_{t}^{k+1}= & b_{t}^{N}+c_{t}^{N} \sum_{j=k+1}^{N-1} \mathrm{D} f^{j}\left(t, X_{t-}\right) \\
& +\int_{\mathbb{R}^{d}}\left(\prod_{j=k+1}^{N-1} \mathrm{e}^{f^{j}\left(t, X_{t-}+x\right)-f^{j}\left(t, X_{t-}\right)}-1\right) x F_{t}^{N}(\mathrm{~d} x) \\
c_{t}^{k+1}= & c_{t}^{N} \\
F_{t}^{k+1}(\mathrm{~d} x)= & \prod_{j=k+1}^{N-1} \mathrm{e}^{f^{j}\left(t, X_{t-}+x\right)-f^{j}\left(t, X_{t-}\right)} F_{t}^{N}(\mathrm{~d} x) .
\end{aligned}
$$

Now, the forward price process $F\left(\cdot, T_{k}, T_{k+1}\right)$ with dynamics

$$
F\left(t, T_{k}, T_{k+1}\right)=\mathrm{e}^{f^{k}\left(t, X_{t}\right)}, \quad t \in\left[0, T_{k-1}\right],
$$

is a $\mathbb{P}_{k+1}$-local martingale if the following condition holds

$$
\begin{aligned}
\left\langle\mathrm{D} f^{k}(\right. & \left.\left.t, X_{t-}\right), b^{k+1}\right\rangle \\
= & -\frac{\mathrm{d}}{\mathrm{d} t} f^{k}\left(t, X_{t-}\right)-\frac{1}{2} \sum_{i, j=1}^{d} \mathrm{D}_{i j}^{2} f^{k}\left(t, X_{t-}\right)\left(c_{t}^{k+1}\right)^{i j} \\
& -\frac{1}{2}\left\langle\mathrm{D} f^{k}\left(t, X_{t-}\right), c^{k+1} \mathrm{D} f^{k}\left(t, X_{t-}\right)\right\rangle \\
& -\int_{\mathbb{R}^{d}}\left(\mathrm{e}^{f^{k}\left(t, X_{t-}+x\right)-f^{k}\left(t, X_{t-}\right)}-1-\left\langle\mathrm{D} f^{k}\left(t, X_{t-}\right), x\right\rangle\right) F_{t}^{k+1}(\mathrm{~d} x)
\end{aligned}
$$

cf. Proposition A.1. By replacing (3.9) into (3.10) we see, after some straightforward calculations, that the latter is equivalent to the $(\mathbb{D R I F T})$ condition. We can also verify that conditions (A.4) and (A.5) from Proposition A.2 hold for the function $f^{k}$ that satisfies $(\mathbb{L} \mathbb{I P})$ and the process $X$ that satisfies $(\mathbb{I N T})$. 
Indeed, we have

$$
\int_{0}^{T_{N}}\left\|c_{t}^{k+1}\right\| \mathrm{d} t=\int_{0}^{T_{N}}\left\|c_{t}^{N}\right\| \mathrm{d} t<C_{2},
$$

hence condition (A.5) holds. Moreover, using $(\mathbb{L} \mathbb{P P})$ and $(\mathbb{I N T})$ we get that

$$
\begin{aligned}
& \int_{0}^{T_{N}} \int_{\mathbb{R}^{d}}\left(|x|^{2} \wedge 1\right) F_{t}^{k+1}(\mathrm{~d} x) \mathrm{d} t+\int_{0}^{T_{N}} \int_{|x|>1}|x| \mathrm{e}^{K^{k}|x|} F_{t}^{k+1}(\mathrm{~d} x) \mathrm{d} t \\
& =\int_{0}^{T_{N}} \int_{\mathbb{R}^{d}}\left(|x|^{2} \wedge 1\right) \prod_{j=k+1}^{N-1} \mathrm{e}^{f^{j}\left(t, X_{t-}+x\right)-f^{j}\left(t, X_{t-}\right)} F_{t}^{N}(\mathrm{~d} x) \mathrm{d} t \\
& \quad+\int_{0}^{T_{N}} \int_{|x|>1}|x| \mathrm{e}^{K^{k-1}|x|} \prod_{j=k+1}^{N-1} \mathrm{e}^{f^{j}\left(t, X_{t-}+x\right)-f^{j}\left(t, X_{t-}\right)} F_{t}^{N}(\mathrm{~d} x) \mathrm{d} t \\
& \leq \int_{0}^{T_{N}} \int_{\mathbb{R}^{d}}\left\{\left(|x|^{2} \wedge 1\right) \mathrm{e}^{\sum_{j=k+1}^{N-1} K^{j}|x|}+1_{\{|x|>1\}}|x| \mathrm{e}^{\sum_{j=k}^{N-1} K^{j}|x|}\right\} F_{t}^{N}(\mathrm{~d} x) \mathrm{d} t \\
& \leq \text { const } \cdot \int_{0}^{T_{N}}\left\{|x|^{2} 1_{\{|x| \leq 1\}}+|x| \mathrm{e}^{K|x|} 1_{\{|x|>1\}}\right\} F_{t}^{N}(\mathrm{~d} x) \mathrm{d} t \\
& <C_{1},
\end{aligned}
$$

where the second to last inequality holds because the exponential function is bounded in the unit hypercube and the Lipschitz constants are positive. Hence, condition (A.4) holds as well. Thus, Proposition A.2 yields that the process $f^{k}(\cdot, X)$ is exponentially special and the forward price process $F\left(\cdot, T_{k}, T_{k+1}\right)$ is a $\mathbb{P}_{k+1}$-uniformly integrable martingale.

Therefore, exactly as in the previous steps we can use the $\mathbb{P}_{k+1}$-uniformly integrable martingale $f^{k}(\cdot, X)$ to define the measure $\mathbb{P}_{k}$ via

$$
\left.\frac{\mathrm{d} \mathbb{P}_{k}}{\mathrm{~d} \mathbb{P}_{k+1}}\right|_{\mathcal{F} .}=\frac{F\left(\cdot, T_{k}, T_{k+1}\right)}{F\left(0, T_{k}, T_{k+1}\right)}=\frac{\mathrm{e}^{f^{k}(\cdot, X)}}{\mathrm{e}^{f^{k}\left(0, X_{0}\right)}}
$$

Then, we can compute the $\mathbb{P}_{k}$-characteristics of $X$ using Lemma A.4 and consider the 'next' forward price process with dynamics

$$
F\left(\cdot, T_{k-1}, T_{k}\right)=\mathrm{e}^{f^{k-1}\left(\cdot, X^{k-1}\right)} .
$$

This procedure produces an arbitrage-free semimartingale model for the forward price process, and thus also for the LIBOR rate, if the $(\mathbb{D R} \mathbb{R} \mathbb{F} \mathbb{T})$ condition holds for each $k \in \overline{\mathcal{K}}$.

Finally, we can easily show that the measures $\mathbb{P}_{k}$ are indeed forward measures, i.e. that $B\left(\cdot, T_{l}\right) / B\left(\cdot, T_{k}\right)$ is a $\mathbb{P}_{k}$-martingale for all $1 \leq k, l \leq N$. This follows directly from Proposition III.3.8 in Jacod and Shiryaev (2003), using that

$$
\frac{B\left(\cdot, T_{l}\right)}{B\left(\cdot, T_{k}\right)} \frac{\mathrm{d} \mathbb{P}_{k}}{\mathrm{dP}_{l+1}}=\mathrm{e}^{f^{l}(\cdot, X)}
$$


which is a $\mathbb{P}_{l+1}$-martingale.

Remark 3.3. Let us point out that, although the true martingale property of the forward price process is not necessary to guarantee the absence of arbitrage, it is required in order to define the forward measures and to construct the model via backward induction. Aside from this, forward measures play a crucial role in term structure models since they allow to derive tractable formulas for interest rate derivatives. Indeed, the major advantage of forward measures for derivative pricing is that we can avoid the numerical computation of multidimensional integrals over joint distributions.

Remark 3.4. We may assume, if desired, that $d \geq N-1$ in order to ensure there are at least as many driving factors as the number of forward price processes. Moreover, by suitable choices of the functions $f^{k}$ we may select the components of $X$ driving a certain forward price process. See, for example, Section 4.1 where we work with an $N$-1-dimensional process $X$ and set $f^{k}(x)=$ $\hat{f}^{k}\left(x_{k}\right)$, for $x=\left(x_{1}, \ldots, x_{N-1}\right)$ and $\hat{f}^{k}: \mathbb{R} \rightarrow \mathbb{R}$, i.e. each forward price process is driven by a different component of the process $X$.

3.2. Modeling rates under the terminal measure. Another possibility for constructing a model for the forward LIBOR rates, or equivalently the forward price processes, is to start with the family of forward price processes with respect to the terminal bond price $B\left(\cdot, T_{N}\right)$ in the tenor structure, i.e.

$$
F\left(\cdot, T_{k}, T_{N}\right)=\frac{B\left(\cdot, T_{k}\right)}{B\left(\cdot, T_{N}\right)}
$$

for all $k \in \overline{\mathcal{K}}$, and to model them simultaneously under the same measure, typically the terminal forward measure $\mathbb{P}_{N}$. Similarly to the previous section, the construction is based on specifying exponential semimartingale dynamics for the forward price process of the following functional form

$$
F\left(\cdot, T_{k}, T_{N}\right)=\mathrm{e}^{g^{k}\left(\cdot, X^{k}\right)},
$$

where $g^{k}$ are suitable functions and $X^{k}$ are $d$-dimensional semimartingales, for $k \in \overline{\mathcal{K}}$.

Consider a collection of $\mathbb{R}^{d}$-valued semimartingales $X^{k}=\left(X_{t}^{k}\right)_{0 \leq t \leq T_{N}}$ on $\left(\Omega, \mathcal{F}, \mathbb{F}, \mathbb{P}_{N}\right)$ and a collection of functions $g^{k}:\left[0, T_{N}\right] \times \mathbb{R}^{d} \rightarrow \mathbb{R}$ for all $k \in \overline{\mathcal{K}}$, which satisfy the following assumptions:

$\left(\mathbb{L} \mathbb{I} \mathbb{P}^{\prime}\right)$ The function $g^{k}$ belongs to $C^{1,2}\left(\left[0, T_{N}\right] \times \mathbb{R}^{d}\right)$ and is globally Lipschitz, i.e.

$$
\left|g^{k}(t, x)-g^{k}(t, y)\right| \leq \tilde{K}^{k}|x-y|,
$$

for every $t \geq 0$ and any $x, y \in \mathbb{R}^{d}$, where $\tilde{K}^{k}>0$ is a constant.

$\left(\mathbb{I N T ^ { \prime }}\right)$ The process $X^{k}$ is an $\mathbb{R}^{d}$-valued semimartingale with absolutely continuous characteristics $\left(b^{k, N}, c^{k, N}, F^{k, N}\right)$ under $\mathbb{P}_{N}$, such that the following conditions hold

$$
\int_{0}^{T_{N}} \int_{\mathbb{R}^{d}}\left\{|x|^{2} 1_{\{|x| \leq 1\}}+|x| \mathrm{e}^{\tilde{K}^{k}|x|} 1_{\{|x|>1\}}\right\} F_{t}^{k, N}(\mathrm{~d} x) \mathrm{d} t<\tilde{C}_{1}^{k}
$$


and

$$
\int_{0}^{T_{N}}\left\|c_{t}^{k, N}\right\| \mathrm{d} t<\tilde{C}_{2}^{k},
$$

for some constants $\tilde{C}_{1}^{k}, \tilde{C}_{2}^{k}>0$. Recall that the truncation can be chosen the identity.

Theorem 3.5. Consider $\mathbb{R}^{d}$-valued semimartingales $X^{k}$ and functions $g^{k}$ such that Assumptions $\left(\mathbb{L} \mathbb{I P} \mathbb{P}^{\prime}\right)$ and $\left(\mathbb{N N}^{\prime}\right)$ are satisfied for each $k \in \overline{\mathcal{K}}$. Assume that the forward price processes are modeled via

$$
F\left(t, T_{k}, T_{N}\right)=\mathrm{e}^{g^{k}\left(t, X_{t}^{k}\right)}, \quad t \in\left[0, T_{k}\right],
$$

and the following drift condition is satisfied

$$
\begin{aligned}
\left\langle\mathrm{D} g^{k}\right. & \left.\left(t, X_{t-}^{k}\right), b_{t}^{k, N}\right\rangle \\
= & -\frac{\mathrm{d}}{\mathrm{d} t} g^{k}\left(t, X_{t-}^{k}\right)-\frac{1}{2} \sum_{i, j=1}^{d} \mathrm{D}_{i j}^{2} g^{k}\left(t, X_{t-}^{k}\right)\left(c_{t}^{k, N}\right)^{i j} \\
& -\frac{1}{2}\left\langle\mathrm{D} g^{k}\left(t, X_{t-}^{k}\right), c_{t}^{k, N} \mathrm{D} g^{k}\left(t, X_{t-}^{k}\right)\right\rangle \\
& -\int_{\mathbb{R}^{d}}\left(\mathrm{e}^{g^{k}\left(t, X_{t-}^{k}+x\right)-g^{k}\left(t, X_{t-}^{k}\right)}-1-\left\langle\mathrm{D} g^{k}\left(t, X_{t-}^{k}\right), x\right\rangle\right) F_{t}^{k, N}(\mathrm{~d} x),
\end{aligned}
$$

for all $k \in \overline{\mathcal{K}}$. Then, the forward price processes are uniformly integrable martingales with respect to the terminal forward measure $\mathbb{P}_{N}$, for all $k \in \overline{\mathcal{K}}$. In particular, the model is arbitrage-free and satisfies Axioms $(\mathbb{A} 1)$ and $(\mathbb{A} 2)$, as well as Property $(\mathbb{B} 1)$.

Proof. The proof is simpler compared to the proof of Theorem 3.2 because we work only under the terminal measure $\mathbb{P}_{N}$. Furthermore, we can work simultaneously with all forward price processes $F\left(\cdot, T_{k}, T_{N}\right)$ for each $k \in \overline{\mathcal{K}}$. More precisely, for all $k \in \overline{\mathcal{K}}$ the function $g^{k}$ satisfies $\left(\mathbb{L}_{\mathbb{P}} \mathbb{P}^{\prime}\right)$ and the process $X^{k}$ satisfies $\left(\mathbb{I N T}^{\prime}\right)$, hence the process $g^{k}\left(\cdot, X^{k}\right)$ is an exponentially special semimartingale by Proposition A.2. Using Proposition A.1, we get by virtue of the $\left(\mathbb{D R} \mathbb{R} \mathbb{T}^{\prime}\right)$ condition that $F\left(\cdot, T_{k}, T_{N}\right)$ is a $\mathbb{P}_{N}$-local martingale. Moreover, Proposition A.2 yields that $F\left(\cdot, T_{k}, T_{N}\right)$ is actually a $\mathbb{P}_{N \text {-uniformly integrable }}$ martingale.

Remark 3.6. In this construction we can use a family of semimartingales $X^{k}$, $k \in \overline{\mathcal{K}}$, where each forward price process is driven by a different semimartingale. This is possible because we do not have to perform measure changes as we did in the backward construction, since all forward price processes are modeled under a common measure. Hence, at this stage, we do not need to know the dependence structure between the processes $X^{k}$ which is necessary when applying Girsanov's theorem. However, for pricing purposes and also for linking the backward and the terminal measure constructions, we revert to a common $\mathbb{R}^{d}$-valued driving process $X$ for which the dependence structure between its components is obviously fully known. Naturally, the dimension of the process $X$ can be chosen such that each rate is driven by a different component of the process; compare with Remark 3.4. 
Remark 3.7. Based on (3.11), we can immediately deduce the dynamics of the forward price process $F\left(\cdot, T_{k}, T_{k+1}\right)$ and the forward LIBOR rate $L\left(\cdot, T_{k}\right)$, for all $k \in \overline{\mathcal{K}}$. Using that

$$
1+\delta L\left(\cdot, T_{k}\right)=F\left(\cdot, T_{k}, T_{k+1}\right)=\frac{F\left(\cdot, T_{k}, T_{N}\right)}{F\left(\cdot, T_{k+1}, T_{N}\right)},
$$

we obtain that

$$
1+\delta L\left(\cdot, T_{k}\right)=F\left(\cdot, T_{k}, T_{k+1}\right)=\mathrm{e}^{g^{k}\left(\cdot, X^{k}\right)-g^{k+1}\left(\cdot, X^{k+1}\right)} .
$$

Remark 3.8. Assumptions $\left(\mathbb{L} \mathbb{P} \mathbb{P}^{\prime}\right)$ and $\left(\mathbb{I N \mathbb { T } ^ { \prime }}\right)$ are sufficient to produce an arbitrage-free family of LIBOR rates, but they are by no means necessary. Indeed, we can weaken them slightly by assuming that the functions $g^{k}$ satisfy $\left(\mathbb{L} \mathbb{I} \mathbb{P}^{\prime}\right)$ and the processes $X^{k}$ have finite exponential moments. Then the previous theorem yields an arbitrage-free model that satisfies Axioms $(\mathbb{A} 1)$ and $(\mathbb{A} 2)$, but not necessarily (B1). However, as pointed out also in Remark 3.3, the latter is needed to define forward measures which are very useful because they typically lead to tractable pricing formulas.

Remark 3.9. Let us consider the case where all semimartigales $X^{k}$ coincide, i.e. $X^{k} \equiv X$ for all $k \in \mathcal{K}$. Then, we can easily link the approach using backward induction presented in subsection 3.1 and the approach under the terminal measure presented in this subsection. More precisely, starting from a family of functions $g^{k}, k \in \overline{\mathcal{K}}$, and a semimartingale $X$ satisfying $(\mathbb{L} \mathbb{I P}),\left(\mathbb{I N T ^ { \prime }}\right)$ and $\left(\mathbb{D R} \mathbb{R} \mathbb{F}^{\prime}\right)$, we define

$$
f^{k}(t, x):=g^{k}(t, x)-g^{k+1}(t, x) .
$$

The functions $f^{k}$ obviously satisfy $(\mathbb{L} \mathbb{P})$ with the constants $K^{k}:=\tilde{K}^{k}+\tilde{K}^{k+1}$. Assume moreover that the semimartingale $X$ satisfies $(\mathbb{I N T})$ with $K^{k}$ as above. Then the model for the terminal forward prices given by (3.11) can be equivalently written as

$$
F\left(\cdot, T_{k}, T_{k+1}\right)=\mathrm{e}^{f^{k}(\cdot, X)}, \quad k \in \overline{\mathcal{K}},
$$

with $f^{k}$ given by (3.16) and all assertions of Theorem 3.2 remain valid.

Conversely, assuming that a model for the forward prices (3.5) is given via a family of functions $f^{k}, k \in \overline{\mathcal{K}}$, and a semimartingale $X$ satisfying $(\mathbb{L I P}),(\mathbb{I N T})$ and $(\mathbb{D} \mathbb{R} \mathbb{N} \mathbb{F})$, we define

$$
g^{k}(t, x):=\sum_{j=k}^{N-1} f^{j}(t, x)
$$

The functions $g^{k}$ satisfy condition $(\mathbb{L} \mathbb{I P})$ with the constants $\tilde{K}^{k}:=\sum_{j=k}^{N-1} K^{j}$. Assuming furthermore that the semimartingale $X$ satisfies $\left(\mathbb{N} \mathbb{N}^{\prime}\right)$ with $\tilde{K}^{k}$ as above, the model for the forward prices (3.5) can be equivalently written as

$$
F\left(\cdot, T_{k}, T_{N}\right)=\mathrm{e}^{g^{k}(\cdot, X)}, \quad k \in \overline{\mathcal{K}},
$$

with $g^{k}$ defined in (3.17). This easily follows from the following telescopic product

$$
F\left(\cdot, T_{k}, T_{N}\right)=\frac{B\left(\cdot, T_{k}\right)}{B\left(\cdot, T_{N}\right)}=\prod_{j=k}^{N-1} \frac{B\left(\cdot, T_{j}\right)}{B\left(\cdot, T_{j+1}\right)}=\prod_{j=k}^{N-1} F\left(\cdot, T_{j}, T_{j+1}\right) .
$$


Thus, we conclude that Theorem 3.5 is valid for the semimartingale $X$ and the functions $g^{k}, k \in \overline{\mathcal{K}}$.

3.3. Observations and ramifications. Next, we discuss further properties of the models constructed in the previous two subsections. In particular, we derive conditions such that a LIBOR model is structure preserving and produces non-negative rates. In order to provide a unified treatment of both modeling approaches, we assume that $X^{k} \equiv X$ in subsection 3.2 , for all $k \in \overline{\mathcal{K}}$.

Lemma 3.10. (i) If the functions $f^{k}$ are non-negative for all $k \in \overline{\mathcal{K}}$, then the LIBOR rates in the model (3.5) are non-negative, i.e. Property $(\mathbb{C})$ is satisfied.

(ii) If the functions $g^{k}$ are non-negative and such that $g^{k}(t, x) \geq g^{k+1}(t, x)$ for all $k \in \overline{\mathcal{K}}$ and all $(t, x) \in\left[0, T_{N}\right] \times \mathbb{R}^{d}$, then the LIBOR rates in the model (3.14) are non-negative, i.e. Property $(\mathbb{C})$ is satisfied.

Proof. This follows directly from the relation between forward prices and LIBOR rates, see (2.3) and (3.15).

The second tractability property $(\mathbb{B} 2)$ states that a LIBOR model is structure preserving if the characteristics of the driving process are transformed in a deterministic way under different forward measures, which ensures that the driving processes remain in the same class under all forward measures. In order to formalize the statement, we consider the following assumption.

$(\mathbb{E})$ Let $U:=\mathbb{R}^{d}$ (respectively $U:=\mathbb{R}_{+}^{d}$ ). The measure $\mathbb{P}_{N}^{X_{t-}}$ is absolutely continuous with positive Lebesgue density on $U$, for all $t \in\left[0, T_{N}\right]$.

We say that a LIBOR model is structure preserving if the tuple $\left(\beta^{l}, Y^{l}\right)$ defining the change of measure from the forward measure $\mathbb{P}_{l}$ to $\mathbb{P}_{l-1}$, for $l=N, \ldots, 1$, via Girsanov's theorem as in Lemma A.4, is deterministic.

Notice that under assumption $(\mathbb{E}), \beta^{l}$ is deterministic if and only if $Y^{l}$ is so; indeed, if $Y^{l}(t, x)=\mathrm{e}^{f^{l}\left(t, X_{t-}+x\right)-f^{l}\left(t, X_{t-}\right)}$ is assumed to be deterministic, the function $f^{l}$ must satisfy

$$
f^{l}(t, y+x)-f^{l}(t, y)=h^{l}(t, x)
$$

for every $x, y \in U$, for some function $h^{l}$. Taking derivatives with respect to $y$, we get that

$$
\mathrm{D} f^{l}(t, y+x)=\mathrm{D} f^{l}(t, y)
$$

for every $x, y \in U$, hence $\mathrm{D} f^{l}(y)$ is constant, and thus $\left.f^{l}(t, \cdot)\right|_{U}$ is an affine function. This implies that $\beta_{t}^{l}=\mathrm{D} f^{l}\left(t, X_{t-}\right)$ is deterministic.

Conversely, assume that the variable $\beta_{t}^{l}=\mathrm{D} f^{l}\left(t, X_{t-}\right)$ is deterministic. Since the support of $\mathbb{P}_{N}^{X_{t-}}$ is $U, \mathrm{D} f^{l}$ is continuous and $\mathbb{P}_{N}^{X_{t-}}$ has a positive Lebesgue measure on $U$, we obtain that $\mathrm{D} f^{l}$ is constant and hence $f^{l}$ is affine in the second variable. Thus, we conclude that $Y^{l}(t, x)$ is deterministic.

The next result provides necessary and sufficient conditions for $(\mathbb{B} 2)$ to be satisfied.

Proposition 3.11. If the functions $f^{k}$ and $g^{k}$ are affine in the second variable for every $k \in \overline{\mathcal{K}}$, then the LIBOR models in (3.5) and (3.14) are structure preserving. Conversely, assume $(\mathbb{E})$. If the LIBOR models in (3.5) and (3.14) are structure preserving, then the functions $\left.f^{k}\right|_{\left[0, T_{N}\right] \times U}$ and $\left.g^{k}\right|_{\left[0, T_{N}\right] \times U}$ are affine in the second variable for every $k \in \overline{\mathcal{K}}$. 
Proof. We will concentrate on the model constructed by backward induction, while the other one follows analogously. Following the argumentation in the proof of Theorem 3.2, the characteristics of $X$ under $\mathbb{P}_{l}$ have the following form

$$
\begin{aligned}
b_{t}^{l}= & b_{t}^{N}+c_{t}^{N} \sum_{j=l+1}^{N-1} \mathrm{D} f^{j}\left(t, X_{t-}\right) \\
& +\int_{\mathbb{R}^{d}}\left(\prod_{j=l+1}^{N-1} \mathrm{e}^{f^{j}\left(t, X_{t-}+x\right)-f^{j}\left(t, X_{t-}\right)}-1\right) x F_{t}^{N}(\mathrm{~d} x) \\
c_{t}^{l}= & c_{t}^{N} \\
F_{t}^{l}(\mathrm{~d} x)= & \prod_{j=l+1}^{N-1} \mathrm{e}^{f^{j}\left(t, X_{t-}+x\right)-f^{j}\left(t, X_{t-}\right)} F_{t}^{N}(\mathrm{~d} x) .
\end{aligned}
$$

Assume that the function $f^{k}(t, x)$ is affine in $x$, i.e. there exist $\alpha^{k}(t)$ and $\beta^{k}(t)$ such that $f^{k}(t, x)=\alpha^{k}(t)+\left\langle\beta^{k}(t), x\right\rangle$, then we can easily deduce that $\left(b^{l}, c^{l}, F^{l}\right)$ in (3.20) is only a deterministic transformation of $\left(b^{N}, c^{N}, F^{N}\right)$.

The converse statement is already implied by the arguments preceding this Proposition.

The statement of Proposition 3.11 can be generalized to allow for more general driving processes. Assumption $(\mathbb{E})$, for instance, can be formulated for more general sets $U$. As an example, $X$ could be a process that is positive in some coordinate and real or negative in another. On the other hand, processes with fixed jump sizes, such as the Poisson process, require a slightly different approach than in the proof above, taking care of the state space of the process and the support of the jump measure.

The following remark summarizes further interesting properties of LIBOR rates that can be easily deduced from this general modeling framework.

Remark 3.12. If the function $f^{k}$ is affine in the second argument, i.e.

$$
f^{k}(t, x)=\alpha^{k}(t)+\left\langle\beta^{k}(t), x\right\rangle,
$$

with functions $\alpha^{k}, \beta^{k} \in C^{1}\left(\mathbb{R}_{+}\right)$and the process is required to satisfy

$$
F\left(\cdot, T_{k}, T_{k+1}\right) \geq 1,
$$

i.e. produce non-negative LIBOR rates, then the process $X$ has to be bounded from below.

\section{EXAMPLES}

4.1. LIBOR market models. We start by revisiting the class of LIBOR market models in view of the general framework developed in the previous section. We will concentrate on the Lévy LIBOR model of Eberlein and Özkan (2005) in order to fix ideas and processes, and as a representative of other LIBOR market models which fit in this framework as well, such as models with local volatility, stochastic volatility or driven by jump-diffusions. See, among many other references, Brigo and Mercurio (2006), Schoenmakers (2005), Glasserman (2003) and Andersen and Piterbarg (2010). 
We assume that the driving process $X$ is an $\mathbb{R}^{N-1}$-valued semimartingale of the form

$$
X=B+\Lambda \cdot L,
$$

where $L$ is an $\mathbb{R}^{n}$-valued time-inhomogeneous Lévy process with characteristic triplet $\left(0, c^{L}, F^{L}\right)$ under the terminal measure $\mathbb{P}_{N}$ with respect to the truncation function $h(x)=x$, and $\Lambda=\left[\lambda\left(\cdot, T_{1}\right), \ldots, \lambda\left(\cdot, T_{N-1}\right)\right]$ is an $(N-1) \times n$ volatility matrix where, for every $k \in \overline{\mathcal{K}}, \lambda\left(\cdot, T_{k}\right)$ is a deterministic, $n$-dimensional function. Moreover, $\Lambda \cdot L$ denotes the Itô stochastic integral of $\Lambda$ with respect to $L$, while the drift term $B=\int_{0}^{\dot{b}} b(s) \mathrm{d} s=\left(\int_{0}^{*} b\left(s, T_{1}\right) \mathrm{d} s, \ldots, \int_{0}^{\dot{b}} b\left(s, T_{N-1}\right) \mathrm{d} s\right)$ is an $(N-1)$-dimensional stochastic process. We further assume that the following exponential moment condition is satisfied:

$(\mathbb{E M})$ Let $\varepsilon>0$ and $M>0$, then

$$
\int_{0}^{T_{N}} \int_{|x|>1} \mathrm{e}^{\langle u, x\rangle} F_{s}^{L}(\mathrm{~d} x) \mathrm{d} s<\infty \quad \text { for all } u \in[-(1+\varepsilon) M,(1+\varepsilon) M]^{n} ;
$$

while the volatility functions satisfy:

$(\mathbb{V} \mathbb{O L})$ The volatility $\lambda\left(\cdot, T_{k}\right):\left[0, T_{N}\right] \rightarrow \mathbb{R}_{+}^{n}$ is a deterministic, bounded function such that for $s>T_{k}, \lambda\left(s, T_{k}\right)=0$, for every $k \in \overline{\mathcal{K}}$. Moreover,

$$
\sum_{k=1}^{N} \lambda^{j}\left(s, T_{k}\right) \leq M \quad \text { for all } s \in\left[0, T_{N}\right]
$$

for every $s \in\left[0, T_{N}\right]$ and every coordinate $j \in\{1, \ldots, n\}$.

The construction of the Lévy LIBOR model will follow the backward induction approach of subsection 3.1. Define, for all $k \in \overline{\mathcal{K}}$ and $x=\left(x_{1}, \ldots, x_{N-1}\right) \in$ $\mathbb{R}^{N-1}$, the functions

$$
f^{k}(t, x):=\log \left(1+\delta_{k} L\left(0, T_{k}\right) \mathrm{e}^{x_{k}}\right)
$$

and set

$$
F\left(t, T_{k}, T_{k+1}\right)=\mathrm{e}^{f^{k}\left(t, X_{t}\right)}, \quad k \in \overline{\mathcal{K}} .
$$

Then, it follows easily that

$$
F\left(t, T_{k}, T_{k+1}\right)=1+\delta_{k} L\left(0, T_{k}\right) \mathrm{e}^{X_{t}^{k}},
$$

which coincides with the dynamics of the Lévy LIBOR model of Eberlein and Özkan (2005), that are provided by

$$
L\left(t, T_{k}\right)=L\left(0, T_{k}\right) \exp \left(\int_{0}^{t} b\left(s, T_{k}\right) \mathrm{d} s+\int_{0}^{t} \lambda\left(s, T_{k}\right) \mathrm{d} L_{s}\right) .
$$

The function $f^{k}$ is Lipschitz continuous with constant 1 , hence condition $(\mathbb{L} \mathbb{I P})$ is satisfied with $K^{k}=1$ for each $k \in \overline{\mathcal{K}}$. Moreover, thanks to assumptions $(\mathbb{E M})$ and $(\mathbb{V} \mathbb{O L})$, condition $(\mathbb{I N T})$ is also satisfied for every $k \in \overline{\mathcal{K}}$. Therefore, an application of Theorem 3.2 yields the drift $B^{k}=\int_{0}^{*} b\left(s, T_{k}\right) \mathrm{d} s$ of this model under the terminal measure. More precisely, we have that

$$
\partial_{x_{k}} f^{k}(t, x)=\frac{\delta_{k} L\left(0, T_{k}\right) \mathrm{e}^{x_{k}}}{1+\delta_{k} L\left(0, T_{k}\right) \mathrm{e}^{x_{k}}}=: \ell^{k}\left(x_{k}\right),
$$


and $\partial_{x_{j}} f^{k}(t, x)=0$, for $j \neq k$, while also $\frac{\mathrm{d}}{\mathrm{d} t} f^{k}(t, x)=0$. Moreover,

$$
\partial_{x_{k} x_{k}} f^{k}(t, x)=\frac{\delta_{k} L\left(0, T_{k}\right) \mathrm{e}^{x_{k}}}{\left(1+\delta_{k} L\left(0, T_{k}\right) \mathrm{e}^{x_{k}}\right)^{2}}
$$

and $\partial_{x_{i} x_{j}} f^{k}(t, x)=0$, for all $(i, j) \neq(k, k)$. According to Proposition 2.4 in Kallsen (2006), the $\mathbb{P}_{N^{-}}$-characteristics $\left(b^{N}, c^{N}, F^{N}\right)$ of $X$ are given by

$$
\begin{aligned}
b_{t}^{N} & =b(t) \\
c_{t}^{N} & =\left\langle\Lambda(t), c_{t}^{L} \Lambda(t)\right\rangle \\
F_{t}^{N}(A) & =\int_{\mathbb{R}^{n}} 1_{A}(\Lambda(t) x) F_{t}^{L}(\mathrm{~d} x), \quad A \in \mathcal{B}\left(\mathbb{R}^{N-1}\right) \backslash\{0\},
\end{aligned}
$$

hence the $(\mathbb{D R} \mathbb{R} \mathbb{F} \mathbb{T})$ condition from Theorem 3.2 becomes

$$
\begin{aligned}
\ell^{k}\left(X_{t-}^{k}\right) b\left(t, T_{k}\right) & =-\frac{1}{2} \frac{\ell^{k}\left(X_{t-}^{k}\right)}{1+\delta_{k} L\left(0, T_{k}\right) \mathrm{e}^{X_{t-}^{k}}}\left\langle\lambda\left(t, T_{k}\right), c_{t}^{L} \lambda\left(t, T_{k}\right)\right\rangle \\
& -\frac{1}{2}\left(\ell^{k}\left(X_{t-}^{k}\right)\right)^{2}\left\langle\lambda\left(t, T_{k}\right), c_{t}^{L} \lambda\left(t, T_{k}\right)\right\rangle \\
& -\sum_{j=k+1}^{N-1} \ell^{k}\left(X_{t-}^{k}\right) \ell^{j}\left(X_{t-}^{j}\right)\left\langle\lambda\left(t, T_{k}\right), c_{t}^{L} \lambda\left(t, T_{j}\right)\right\rangle \\
& -\int_{\mathbb{R}^{N-1}}\left[( \mathrm { e } ^ { f ^ { k } ( X _ { t - } ^ { k } + x _ { k } ) - f ^ { k } ( X _ { t - } ^ { k } ) } - 1 ) \prod _ { j = k + 1 } ^ { N - 1 } \left(\mathrm{e}^{\left.f^{j}\left(X_{t-}^{j}+x_{j}\right)-f^{j}\left(X_{t-}^{k}\right)\right)}\right.\right. \\
& \left.-\ell^{k}\left(X_{t-}^{k} x_{k}\right)\right] F_{t}^{X}(\mathrm{~d} x)
\end{aligned}
$$

Notice that

$$
\begin{aligned}
\frac{\ell^{k}\left(X_{t-}^{k}\right)}{1+\delta_{k} L\left(0, T_{k}\right) \mathrm{e}^{X_{t-}^{k}}}+\left(\ell^{k}\left(X_{t-}^{k}\right)\right)^{2} & =\frac{\delta_{k} L\left(0, T_{k}\right) \mathrm{e}^{X_{t-}^{k}}+\left(\delta_{k} L\left(0, T_{k}\right) \mathrm{e}^{X_{t-}^{k}}\right)^{2}}{\left(1+\delta_{k} L\left(0, T_{k}\right) \mathrm{e}^{X_{t-}^{k}}\right)^{2}} \\
& =\frac{\delta_{k} L\left(0, T_{k}\right) \mathrm{e}^{X_{t-}^{k}}}{1+\delta_{k} L\left(0, T_{k}\right) \mathrm{e}^{X_{t-}^{k}}}=\ell\left(X_{t-}^{k}\right)
\end{aligned}
$$

and that, for all $j=k, \ldots, N-1$,

$$
\begin{aligned}
\mathrm{e}^{f^{j}\left(X_{t-}^{j}+x_{j}\right)-f^{j}\left(X_{t-}^{k}\right)} & =\frac{1+\delta_{j} L\left(0, T_{j}\right) \mathrm{e}^{X_{t-}^{j}+x_{j}}}{1+\delta_{j} L\left(0, T_{j}\right) \mathrm{e}^{X_{t-}^{j}}} \\
& =\frac{1+\delta_{j} L\left(0, T_{j}\right) \mathrm{e}^{X_{t-}^{j}}+\delta_{j} L\left(0, T_{j}\right) \mathrm{e}^{X_{t-}^{j}}\left(\mathrm{e}^{x_{j}}-1\right)}{1+\delta_{j} L\left(0, T_{j}\right) \mathrm{e}^{X_{t-}^{j}}} \\
& =1+\ell^{j}\left(X_{t-}^{j}\right)\left(\mathrm{e}^{x_{j}}-1\right) .
\end{aligned}
$$


Inserting the above simplifications into (4.7) yields

$$
\begin{aligned}
b\left(t, T_{k}\right)= & -\frac{1}{2}\left\langle\lambda\left(t, T_{k}\right), c_{t}^{L} \lambda\left(t, T_{k}\right)\right\rangle-\sum_{j=k+1}^{N-1} \ell^{j}\left(X_{t-}^{j}\right)\left\langle\lambda\left(t, T_{k}\right), c_{t}^{L} \lambda\left(t, T_{j}\right)\right\rangle \\
& -\int_{\mathbb{R}^{N}}\left[\left(\mathrm{e}^{x_{k}}-1\right) \prod_{j=k+1}^{N-1}\left(1+\ell^{j}\left(X_{t-}^{j}\right)\left(\mathrm{e}^{x_{j}}-1\right)\right)-x_{k}\right] F_{t}^{X}(\mathrm{~d} x) \\
= & -\frac{1}{2}\left\langle\lambda\left(t, T_{k}\right), c_{t}^{L} \lambda\left(t, T_{k}\right)\right\rangle-\sum_{j=k+1}^{N-1} \ell^{j}\left(X_{t-}^{j}\right)\left\langle\lambda\left(t, T_{k}\right), c_{t}^{L} \lambda\left(t, T_{j}\right)\right\rangle \\
& -\int_{\mathbb{R}^{n}}\left[\left(\mathrm{e}^{\left\langle\lambda\left(t, T_{k}\right), y\right\rangle}-1\right) \prod_{j=k+1}^{N-1}\left(1+\ell^{j}\left(X_{t-}^{j}\right)\left(\mathrm{e}^{\left\langle\lambda\left(t, T_{j}\right), y\right\rangle}-1\right)\right)\right. \\
& \left.-\left\langle\lambda\left(t, T_{k}\right), y\right\rangle\right] F_{t}^{L}(\mathrm{~d} y),
\end{aligned}
$$

where the second equality follows by (4.6). The equation above now can be recognized as the drift condition of the Lévy LIBOR model; cf. Papapantoleon, Schoenmakers, and Skovmand(2012, eq. (2.7)).

Remark 4.1. The LIBOR market models satisfy Axioms $(\mathbb{A} 1)$ and $(\mathbb{A} 2)$, as well as Properties $(\mathbb{B} 1)$ and $(\mathbb{B} 4)$ by construction. On the other hand, Properties $(\mathbb{B} 2)$ and $(\mathbb{B} 3)$ are not satisfied. Regarding $(\mathbb{B} 2)$, this follows immediately by Proposition 3.11 (at least for driving processes satisfying $(\mathbb{E}$ ), which is typically the case), since the functions $f^{k}, k \in \overline{\mathcal{K}}$, are not affine in the second argument. Moreover, the drift term (4.8) which contains the random terms $\delta_{j} L\left(t, T_{j}\right) /(1+$ $\left.\delta_{j} L\left(t, T_{j}\right)\right)$ implies that the vector of LIBOR rates $\left(L\left(\cdot, T_{k}\right)\right)_{k \in \overline{\mathcal{K}}}$ considered as a whole is Markovian, but not the single LIBOR rates, because their dynamics depend on the other rates as well. Hence, (BB3) does not hold. Finally, Property $(\mathbb{C})$ is obviously satisfied in this model.

4.2. Lévy forward price models. Next, we show that the Lévy forward price models can be easily embedded in our general framework starting from the terminal measure construction; starting from the backward induction approach is even easier. The Lévy forward price models were introduced by Eberlein and Özkan (2005, pp. 342-343); see also Kluge (2005) for a detailed construction and Kluge and Papapantoleon (2009) for a concise presentation.

We will model the dynamics of the forward price relative to the terminal bond price under the terminal measure $\mathbb{P}_{N}$, via

$$
F\left(t, T_{k}, T_{N}\right)=\mathrm{e}^{g^{k}\left(t, X_{t}^{k}\right)},
$$

where the function $g^{k}$ is of the following affine form

$$
g^{k}(t, x):=\log F\left(0, T_{k}, T_{N}\right)+x,
$$

while the process $X^{k}$ has the following dynamics

$$
X^{k}:=\int_{0} b_{s}^{k, N} \mathrm{~d} s+\sum_{i=k}^{N-1} \int_{0} \lambda\left(s, T_{i}\right) \mathrm{d} L_{s} .
$$


The driving process $L$ and the volatility functions $\lambda\left(\cdot, T_{i}\right)$ are specified, while the drift term $b^{k, N}$ is determined by the no-arbitrage $\left(\mathbb{D} \mathbb{R} \mathbb{R} \mathbb{F}^{\prime}\right)$ condition. In particular, $L$ is an $\mathbb{R}^{n}$-valued time-inhomogeneous Lévy process with $\mathbb{P}_{N}$-local characteristics $\left(0, c^{L}, F^{L}\right)$ satisfying condition $(\mathbb{E M})$ and the volatility functions satisfy condition $(\mathbb{V} \mathbb{O L})$. The function $g^{k}$ trivially satisfies the $\left(\mathbb{L} \mathbb{P} \mathbb{P}^{\prime}\right)$ condition with constant 1 , while the process $X^{k}$ satisfies the $\left(\mathbb{I N T}^{\prime}\right)$ condition by virtue of $(\mathbb{E M})$ and $(\mathbb{V O L})$; see also Criens, Glau, and Grbac (2015, Remark 3.7). Therefore, we can apply Theorem 3.5 and, after some computations, the $\left(\mathbb{D} \mathbb{R} \mathbb{N} \mathbb{T}^{\prime}\right)$ condition yields that

$$
b_{t}^{k, N}=-\frac{1}{2} c_{t}^{k, N}-\int_{\mathbb{R}}\left(\mathrm{e}^{x}-1-x\right) F_{t}^{k, N}(\mathrm{~d} x) .
$$

Moreover, using Kallsen and Shiryaev (2002b, Lemma 3), the $\mathbb{P}_{N^{-}}$local characteristics of the stochastic integral process $X^{k}$ are

$$
c_{t}^{k, N}=\left\langle\sum_{i=k}^{N-1} \lambda\left(t, T_{i}\right), c_{t}^{L} \sum_{i=k}^{N-1} \lambda\left(t, T_{i}\right)\right\rangle
$$

and

$$
F_{t}^{k, N}(A)=\int_{\mathbb{R}^{n}} 1_{A}\left(\sum_{i=k}^{N-1}\left\langle\lambda\left(t, T_{i}\right), x\right\rangle\right) F_{t}^{L}(\mathrm{~d} x), \quad A \in \mathcal{B}(\mathbb{R}) .
$$

Now, using (4.9)-(4.11), we get that the dynamics of the forward price process $F\left(\cdot, T_{k}, T_{k+1}\right)$ are provided by

$$
\begin{aligned}
F\left(t, T_{k}, T_{k+1}\right) & =\frac{F\left(t, T_{k}, T_{N}\right)}{F\left(t, T_{k+1}, T_{N}\right)}=F\left(0, T_{k}, T_{k+1}\right) \mathrm{e}^{X_{t}^{k}-X_{t}^{k+1}} \\
& =F\left(0, T_{k}, T_{k+1}\right) \exp \left(\int_{0}^{t}\left(b_{s}^{k, N}-b_{s}^{k+1, N}\right) \mathrm{d} s+\int_{0}^{t} \lambda\left(s, T_{k}\right) \mathrm{d} L_{s}\right),
\end{aligned}
$$

hence the forward price process is driven by its corresponding volatility function and the time-inhomogeneous Lévy process, as specified in the Lévy forward process models. We just have to check that the drift terms coincide as well. Indeed, using (4.12)-(4.14), after some straightforward calculations we get that

$$
\begin{aligned}
b_{s}^{k, N}-b_{s}^{k+1, N} & =-\frac{1}{2}\left\langle\lambda\left(s, T_{k}\right), c_{s}^{L} \lambda\left(s, T_{k}\right)\right\rangle-\sum_{i=k+1}^{N-1}\left\langle\lambda\left(s, T_{k}\right), c_{s}^{L} \lambda\left(s, T_{i}\right)\right\rangle \\
& -\int_{\mathbb{R}^{n}}\left\{\left(\mathrm{e}^{\left\langle\lambda\left(s, T_{k}\right), x\right\rangle}-1\right) \mathrm{e}^{\sum_{i=k+1}^{N-1}\left\langle\lambda\left(s, T_{i}\right), x\right\rangle}-\left\langle\lambda\left(s, T_{k}\right), x\right\rangle\right\} F_{s}^{L}(\mathrm{~d} x),
\end{aligned}
$$

which is exactly the $\mathbb{P}_{N}$-drift of the forward price process; compare with Kluge and Papapantoleon (2009, eqs. (19)-(21)).

Remark 4.2. The Lévy forward price model satisfies Axioms $(\mathbb{A} 1)$ and $(\mathbb{A} 2)$ as well as Properties $(\mathbb{B} 1)$ and $(\mathbb{B} 4)$ by construction. Moreover, it satisfies Properties $(\mathbb{B} 2)$ and $(\mathbb{B} 3)$; cf. Proposition 3.11. Property $(\mathbb{C})$ is not satisfied however, i.e. the LIBOR rates can become negative; cf. Remark 3.12. 
4.3. Affine LIBOR models. Finally, we examine a class of LIBOR models driven by affine processes, and in particular the affine LIBOR models proposed by Keller-Ressel et al. (2013). Our main reference for the definition and properties of affine processes is Duffie, Filipović, and Schachermayer (2003).

Let $X=\left(X_{t}\right)_{0 \leq t \leq T_{N}}$ be a conservative affine process according to Definitions 2.1 and 2.5 in Duffie et al. (2003) with state space $D=\mathbb{R}_{+}$. We consider a one-dimensional process here only for notational simplicity; the $d$-dimensional case can be treated in exactly the same manner. Moreover, the state space is restricted to the positive half-line following Keller-Ressel et al. (2013), which is necessary in order to produce a model satisfying $(\mathbb{C})$; see also Remark 3.12. We can equally well choose the state space $D=\mathbb{R}$, and then interest rates in the model will also take negative values.

The process $X$ is a semimartingale with absolutely continuous characteristics, and the local characteristics $\left(b^{X}, c^{X}, F^{X}\right)$ of $X$ with respect to the truncation function $h(x):=1 \wedge x$, for $x \in D$, are given as

$$
\begin{aligned}
b_{t}^{X} & =\tilde{b}+\beta X_{t-} \\
c_{t}^{X} & =2 \alpha X_{t-} \\
F_{t}^{X}(\mathrm{~d} \xi) & =F^{1}(\mathrm{~d} \xi)+X_{t_{-}} F^{2}(\mathrm{~d} \xi)
\end{aligned}
$$

for some $\tilde{b}>0, \beta \in \mathbb{R}, \alpha>0$ and Lévy measures $F^{1}$ and $F^{2}$ on $D \backslash\{0\}$ (cf. Theorem 2.12 in Duffie et al. (2003)), with

$$
\tilde{b}:=b+\int_{\xi>0} h(\xi) F^{1}(\mathrm{~d} \xi) .
$$

Affine processes are characterized by the following property of their moment generating function:

$$
\mathbb{E}_{\mathrm{x}}\left[\exp \left(u X_{t}\right)\right]=\exp (\phi(t, u)+\psi(t, u) \mathrm{x}),
$$

for all $(t, u, \mathrm{x}) \in\left[0, T_{N}\right] \times \mathcal{I}_{T} \times D$, where $\mathbb{E}_{\mathrm{x}}$ denotes the expectation with respect to $\mathbb{P}_{\mathrm{x}}$-a probability measure such that $X_{0}=\mathrm{x} \in D, \mathbb{P}_{\mathrm{x}}$-a.s. Moreover, the set $\mathcal{I}_{T}$ is defined by

$$
\mathcal{I}_{T}:=\left\{u \in \mathbb{R}: \mathbb{E}_{\mathrm{x}}\left[\mathrm{e}^{u X_{T_{N}}}\right]<\infty, \text { for all } \mathrm{x} \in D\right\},
$$

while $(\phi, \psi)$ is a pair of deterministic functions $\phi, \psi:\left[0, T_{N}\right] \times \mathcal{I}_{T} \rightarrow \mathbb{R}$. The functions $\phi$ and $\psi$ are given as solutions to generalized Riccati equations (cf. Theorem 2.7 in Duffie et al. (2003)), that is

$$
\begin{aligned}
\partial_{t} \phi(t, u) & =F(\psi(t, u)), & \phi(0, u) & =0 \\
\partial_{t} \psi(t, u) & =R(\psi(t, u)), & & \psi(0, u)=u,
\end{aligned}
$$

where

$$
\begin{aligned}
& F(u)=b u+\int_{\xi>0}\left(\mathrm{e}^{u \xi}-1\right) F^{1}(\mathrm{~d} \xi), \\
& R(u)=\alpha u^{2}+\beta u+\int_{\xi>0}\left(\mathrm{e}^{u \xi}-1-u h(\xi)\right) F^{2}(\mathrm{~d} \xi) .
\end{aligned}
$$

We introduce next the class of affine forward price models, where the forward price is an exponentially-affine function of the driving affine process $X$. In 
particular, we consider the setting of the terminal measure construction of subsection 3.2 with

$$
g^{k}(t, x)=\theta^{k}(t)+\vartheta^{k}(t) x \quad \text { and } \quad X^{k} \equiv X .
$$

The next result shows that the functions $\theta^{k}, \vartheta^{k}$ are solutions to generalized Riccati equations themselves.

Proposition 4.3. Let $X$ be an affine process with values in $D, X_{0}=1$ and satisfying $\left(\mathbb{I N T}^{\prime}\right)$, and $g^{k}, k \in \overline{\mathcal{K}}$, be a collection of functions given by (4.19) where $\theta^{k}, \vartheta^{k}:\left[0, T_{N}\right] \rightarrow \mathbb{R}$ are deterministic functions of class $C^{1}$. Then, the forward price process given by

$$
F\left(t, T_{k}, T_{N}\right)=\mathrm{e}^{\theta^{k}(t)+\vartheta^{k}(t) X_{t}}, \quad t \in\left[0, T_{k}\right],
$$

is a uniformly integrable martingale, for all $k \in \overline{\mathcal{K}}$, if the functions $\theta^{k}$ and $\vartheta^{k}$ satisfy

$$
\begin{aligned}
& \partial_{t} \theta^{k}(t)=-F\left(\vartheta^{k}(t)\right), \\
& \partial_{t} \vartheta^{k}(t)=-R\left(\vartheta^{k}(t)\right),
\end{aligned}
$$

with $F$ and $R$ given by (4.18).

Proof. The process $X$ satisfies $\left(\mathbb{N N}^{\prime}\right)$ by assumption, while the functions $g^{k}$ satisfy $\left(\mathbb{L} \mathbb{P} \mathbb{P}^{\prime}\right)$. Therefore, we can apply Theorem 3.5 and the result follows after straightforward calculations, by inserting the characteristics of $X$ into the $\left(\mathbb{D R} \mathbb{R} \mathbb{F}^{\prime}\right)$ condition and using that

$$
\partial_{t} g^{k}(t, x)=\partial_{t} \theta^{k}(t)+\partial_{t} \vartheta^{k}(t) x, \quad \partial_{x} g^{k}(t, x)=\vartheta^{k}(t), \quad \partial_{x x} g^{k}(t, x)=0 .
$$

Remark 4.4. The affine forward price models given by (4.20) satisfy Axioms $(\mathbb{A} 1)$ and $(\mathbb{A} 2)$ as well as Properties $(\mathbb{B} 1)-(\mathbb{B} 3)$. Property $(\mathbb{B} 4)$ is not satisfied and the model has to be calibrated to the initial term structure, similarly to short rate models. Indeed, notice that the initial forward price $F\left(0, T_{k}, T_{N}\right)$ does not appear in the function $g^{k}(t, x)$, contrary to the previous two examples. ${ }^{1}$ Moreover, these models satisfy Property $(\mathbb{C})$ if and only if the functions $\theta^{k}$ and $\vartheta^{k}$ are non-negative; compare also with Remark 3.12.

The affine LIBOR models introduced by Keller-Ressel et al. (2013) can naturally be embedded in this construction. More precisely, we have the following.

Corollary 4.5. The affine LIBOR models whose dynamics are provided by

$$
F\left(t, T_{k}, T_{N}\right)=\mathbb{E}_{N}\left[\mathrm{e}^{u_{k} X_{T_{N}} \mid \mathcal{F}_{t}}\right]=\mathrm{e}^{\phi\left(T_{N}-t, u_{k}\right)+\psi\left(T_{N}-t, u_{k}\right) X_{t}},
$$

with parameters $u_{k} \in \mathbb{R}_{+}$for $k \in \overline{\mathcal{K}}$, is a special case of the affine forward price models with

$$
\theta^{k}(t):=\phi\left(T_{N}-t, u_{k}\right) \quad \text { and } \quad \vartheta^{k}(t):=\psi\left(T_{N}-t, u_{k}\right),
$$

where $\phi\left(\cdot, u_{k}\right)$ and $\psi\left(\cdot, u_{k}\right)$ are solutions to $(4.17)$.

\footnotetext{
${ }^{1}$ We could, of course, use the following affine function $g^{k}(t, x)=\log F\left(0, T_{k}, T_{N}\right)+\theta^{k}(t)+$ $\vartheta^{k}(t) x$ and the model fits automatically the initial term structure. However, it becomes then difficult to provide models that produce non-negative LIBOR rates.
} 


\section{Appendix A. Semimartingale Characteristics and martingales}

Let $\left(\Omega, \mathcal{F},\left(\mathcal{F}_{t}\right)_{t \in\left[0, T_{*}\right]}, \mathbb{P}\right)$ denote a complete stochastic basis and $T_{*}$ denote a finite time horizon. Let $X$ be an $\mathbb{R}^{d}$-valued semimartingale on this basis whose characteristics are absolutely continuous, i.e. its local characteristics are given by $(b, c, F ; A)$ with $A_{t}=t$, for some truncation function $h$; cf. Jacod and Shiryaev (2003, Prop. II.2.9). Moreover, let $f: \mathbb{R}_{+} \times \mathbb{R}^{d} \rightarrow \mathbb{R}$ be a function of class $C^{1,2}\left(\mathbb{R}_{+} \times \mathbb{R}^{d}\right)$.

The process $f(\cdot, X)$ is a real-valued semimartingale which has again absolutely continuous characteristics. Let us denote its local characteristics by $\left(b^{f}, c^{f}, F^{f}\right)$ for a truncation function $h^{f}$. Then, noting that Itô's formula holds for the function $f \in C^{1,2}\left(\mathbb{R}_{+} \times \mathbb{R}^{d}\right)$ and reasoning as in the proof of Corollary A.6 from Goll and Kallsen (2000), we have that

$$
\begin{aligned}
b_{t}^{f}= & \frac{\mathrm{d}}{\mathrm{d} t} f\left(t, X_{t-}\right)+\left\langle\mathrm{D} f\left(t, X_{t-}\right), b_{t}\right\rangle+\frac{1}{2} \sum_{i, j=1}^{d} \mathrm{D}_{i j}^{2} f\left(t, X_{t-}\right) c_{t}^{i j} \\
& +\int_{\mathbb{R}^{d}}\left(h^{f}\left(f\left(t, X_{t-}+x\right)-f\left(t, X_{t-}\right)\right)-\left\langle\mathrm{D} f\left(t, X_{t-}\right), h(x)\right\rangle\right) F_{t}(\mathrm{~d} x) \\
c_{t}^{f}= & \left\langle\mathrm{D} f\left(t, X_{t-}\right), c_{t} \mathrm{D} f\left(t, X_{t-}\right)\right\rangle \\
F_{t}^{f}(G)= & \int_{\mathbb{R}^{d}} 1_{G}\left(f\left(t, X_{t-}+x\right)-f\left(t, X_{t-}\right)\right) F_{t}(\mathrm{~d} x), \quad G \in \mathcal{B}(\mathbb{R} \backslash\{0\})
\end{aligned}
$$

Proposition A.1. Let $X$ be an $\mathbb{R}^{d}$-valued semimartingale with absolutely continuous characteristics $(b, c, F)$ and let $f: \mathbb{R}_{+} \times \mathbb{R}^{d} \rightarrow \mathbb{R}$ be a function of class $C^{1,2}$ such that the process $Y$ defined by

$$
Y_{t}:=\mathrm{e}^{f\left(t, X_{t}\right)}
$$

is exponentially special. If the following condition holds

$$
\begin{aligned}
\left\langle\mathrm{D} f\left(t, X_{t-}\right), b_{t}\right\rangle= & -\frac{\mathrm{d}}{\mathrm{d} t} f\left(t, X_{t-}\right)-\frac{1}{2} \sum_{i, j=1}^{d} \mathrm{D}_{i j}^{2} f\left(t, X_{t-}\right) c_{t}^{i j} \\
& -\frac{1}{2}\left\langle\mathrm{D} f\left(t, X_{t-}\right), c_{t} \mathrm{D} f\left(t, X_{t-}\right)\right\rangle \\
& -\int_{\mathbb{R}^{d}}\left(\mathrm{e}^{f\left(t, X_{t-}+x\right)-f\left(t, X_{t-}\right)}-1-\left\langle\mathrm{D} f\left(t, X_{t-}\right), h(x)\right\rangle\right) F_{t}(\mathrm{~d} x),
\end{aligned}
$$

then $Y$ is a local martingale.

Proof. The proof follows from Theorem 2.18 in Kallsen and Shiryaev (2002a): set $\theta=1$ and apply the theorem to the semimartingale $f(\cdot, X)$. Indeed, since $f(\cdot, X)$ has absolutely continuous characteristics it is also quasi-left continuous, hence assertions (6) and (1) of Theorem 2.18. yield

$$
K^{f(\cdot, X)}(1)=\widetilde{K}^{f(\cdot, X)}(1)=\int_{0}\left(b_{t}^{f}+\frac{1}{2} c_{t}^{f}+\int_{\mathbb{R}}\left(\mathrm{e}^{x}-1-h^{f}(x)\right) F_{t}^{f}(\mathrm{~d} x)\right) \mathrm{d} t .
$$


By definition of the exponential compensator and Theorem 2.19 in Kallsen and Shiryaev (2002a) it follows that

$$
\mathrm{e}^{f(\cdot, X)-K^{f(\cdot, X)}(1)} \in \mathcal{M}_{\text {loc }} .
$$

Therefore, $\mathrm{e}^{f(\cdot, X)} \in \mathcal{M}_{\text {loc }}$ if and only if $K^{f(\cdot, X)}(1)=0$ up to indistinguishability. Equivalently,

$$
b_{t}^{f}+\frac{1}{2} c_{t}^{f}+\int_{\mathbb{R}}\left(\mathrm{e}^{x}-1-h^{f}(x)\right) F_{t}^{f}(\mathrm{~d} x)=0
$$

for every $t$. Inserting the expressions for $b^{f}, c^{f}$ and $F^{f}$, cf. (A.1), into the above equality yields condition (A.3).

Proposition A.2. Let $X$ be an $\mathbb{R}^{d}$-valued semimartingale with absolutely continuous characteristics $(b, c, F)$ such that

$$
\int_{0}^{T_{*}} \int_{\mathbb{R}^{d}}\left(|x|^{2} \wedge 1\right) F_{t}(\mathrm{~d} x) \mathrm{d} t+\int_{0}^{T_{*}} \int_{|x|>1}|x| \mathrm{e}^{K|x|} F_{t}(\mathrm{~d} x) \mathrm{d} t<C_{1}
$$

and

$$
\int_{0}^{T_{*}}\left\|c_{t}\right\| \mathrm{d} t<C_{2}
$$

for some deterministic constants $C_{1}, C_{2}>0$. Moreover, let $f: \mathbb{R}_{+} \times \mathbb{R}^{d} \rightarrow \mathbb{R}$ be a function of class $C^{1,2}$ and globally Lipschitz, i.e. there exists a constant $K>0$ such that

$$
|f(t, x)-f(t, y)| \leq K|x-y|, \quad t \geq 0, \quad x, y \in \mathbb{R}^{d} .
$$

Then, the process $f(\cdot, X)$ is exponentially special, while the process $Y$ defined by (A.2) and satisfying (A.3) is a uniformly integrable martingale.

Proof. The process $f(\cdot, X)$ is exponentially special if and only if

$$
1_{\{|x|>1\}} \mathrm{e}^{x} * \nu^{f} \in \mathcal{V} .
$$

Hence, it suffices to show that $1_{\{|x|>1\}} \mathrm{e}^{x} * \nu_{T_{*}}^{f}<\infty$, as the integrand is positive. Since $f$ is globally Lipschitz, we have

$$
\begin{aligned}
1_{\{|x|>1\}} \mathrm{e}^{x} * \nu_{T_{*}}^{f} & =\int_{0}^{T_{*}} \int_{|x|>1} \mathrm{e}^{x} F_{t}^{f}(\mathrm{~d} x) \mathrm{d} t \\
& \stackrel{(\mathrm{A} .1)}{=} \int_{0}^{T_{*}} \int_{\mathbb{R}^{d}} 1_{\left\{\left|f\left(t, X_{t-}+x\right)-f\left(t, X_{t-}\right)\right|>1\right\}} \mathrm{e}^{f\left(t, X_{t-}+x\right)-f\left(t, X_{t-}\right)} F_{t}(\mathrm{~d} x) \mathrm{d} t \\
& \leq \int_{0}^{T_{*}} \int_{K|x|>1} \mathrm{e}^{K|x|} F_{t}(\mathrm{~d} x) \mathrm{d} t<\infty,
\end{aligned}
$$

which holds by the Lipschitz property and (A.4). 
Moreover, if $F=\mathrm{e}^{f(\cdot, X)} \in \mathcal{M}_{\text {loc }}$, using Proposition 3.4 in Criens et al. (2015) it is also a uniformly integrable martingale if the following condition holds:

$$
\int_{0}^{T_{*}}\left(c_{t}^{f}+\int_{\mathbb{R}^{d}}\left[\left(|x|^{2} \wedge 1\right)+|x| \mathrm{e}^{x} 1_{\{|x|>1\}}\right] F_{t}^{f}(\mathrm{~d} x)\right) \mathrm{d} t<C^{f},
$$

for some constant $C^{f}>0$. We first check the condition for the diffusion coefficient

$$
\int_{0}^{T_{*}} c_{t}^{f} \mathrm{~d} t=\int_{0}^{T_{*}}\left\langle\mathrm{D} f\left(t, X_{t-}\right), c_{t} \mathrm{D} f\left(t, X_{t-}\right)\right\rangle \mathrm{d} t \leq \int_{0}^{T_{*}}\left\|c_{t}\right\|\left|\mathrm{D} f\left(t, X_{t-}\right)\right|^{2} \mathrm{~d} t<C_{1}^{f},
$$

which follows from (A.5) and the fact that $\mathrm{D} f\left(\cdot, X_{-}\right)$is bounded as a consequence of $f$ being globally Lipschitz. As for the jump part, we have that

$$
\begin{aligned}
& \int_{0}^{T_{*}} \int_{\mathbb{R}}\left(|x|^{2} \wedge 1\right) F_{t}^{f}(\mathrm{~d} x) \mathrm{d} t+\int_{0}^{T_{*}} \int_{|x|>1}|x| \mathrm{e}^{x} F_{t}^{f}(\mathrm{~d} x) \mathrm{d} t \\
& \stackrel{(\mathrm{A} .1)}{=} \int_{0}^{T_{*}} \int_{\mathbb{R}^{d}}\left(\left|f\left(t, X_{t-}+x\right)-f\left(t, X_{t-}\right)\right|^{2} \wedge 1\right) F_{t}(\mathrm{~d} x) \mathrm{d} t \\
& \quad+\int_{0}^{T_{*}} \int_{\mathbb{R}^{d}} 1_{\left\{\left|f\left(t, X_{t-}+x\right)-f\left(t, X_{t-}\right)\right|>1\right\}} \\
& \quad \times\left|f\left(t, X_{t-}+x\right)-f\left(t, X_{t-}\right)\right| \mathrm{e}^{f\left(t, X_{t-}+x\right)-f\left(t, X_{t-}\right)} F_{t}(\mathrm{~d} x) \mathrm{d} t \\
& \leq \int_{0}^{T_{*}} \int_{\mathbb{R}^{d}}\left(K^{2}|x|^{2} \wedge 1\right) F(\mathrm{~d} x) \mathrm{d} t+\int_{0}^{T_{*}} \int_{K|x|>1} K|x| \mathrm{e}^{K|x|} F^{f}(\mathrm{~d} x) \mathrm{d} t<C_{2}^{f},
\end{aligned}
$$

using again the Lipschitz property and (A.4).

Next, we provide the representation of $Y$ as a stochastic exponential.

Lemma A.3. Let $X$ be an $\mathbb{R}^{d}$-valued semimartingale with absolutely continuous characteristics $(B, C, \nu)$ and let $f: \mathbb{R}_{+} \times \mathbb{R}^{d} \rightarrow \mathbb{R}_{+}$be a function of class $C^{1,2}\left(\mathbb{R}_{+} \times \mathbb{R}^{d}\right)$. Define a real-valued semimartingale $Y$ via (A.2). If $Y \in \mathcal{M}_{\mathrm{loc}}$, then it can be written as

$$
Y=\mathcal{E}\left(\mathrm{D} f\left(\cdot, X_{-}\right) \cdot X^{c}+W(\cdot, x) *\left(\mu^{X}-\nu\right)\right),
$$

where $X^{c}$ is the continuous martingale part of $X, \mu^{X}$ is the random measure of jumps of $X$ with compensator $\nu$ and

$$
W(\cdot, x):=\mathrm{e}^{f\left(\cdot, X_{-}+x\right)-f\left(\cdot, X_{-}\right)}-1 .
$$

Proof. Theorem 2.19 in Kallsen and Shiryaev (2002a) yields that

$$
\mathrm{e}^{f(\cdot, X)}=\mathcal{E}\left(f(\cdot, X)^{c}+\left(\mathrm{e}^{x}-1\right) *\left(\mu^{f}-\nu^{f}\right)\right),
$$

using that $f(\cdot, X)$ is quasi-left continuous since $X$ is also quasi-left continuous. Here $f(\cdot, X)^{c}$ denotes the continuous martingale part of $f(\cdot, X)$ and $\mu^{f}$ 
its random measure of jumps. The result now follows using the form of the local characteristics $c^{f}, F^{f}$ of the process $f(\cdot, X)$ in (A.1); see also the proof of Corollary A.6 in Goll and Kallsen (2000).

Lemma A.4. Let $X$ be an $\mathbb{R}^{d}$-valued semimartingale with absolutely continuous characteristics $(b, c, F)$ with respect to the truncation function $h$. Let $f: \mathbb{R}_{+} \times \mathbb{R}^{d} \rightarrow \mathbb{R}_{+}$be a function of class $C^{1,2}\left(\mathbb{R}_{+} \times \mathbb{R}^{d}\right)$ and globally Lipschitz. Assume that conditions (A.3), (A.4) and (A.5) are satisfied.

Define the probability measure $\mathbb{P}^{\prime} \sim \mathbb{P}$ via

$$
\left.\frac{\mathrm{d} \mathbb{P}^{\prime}}{\mathrm{d} \mathbb{P}}\right|_{\mathcal{F} .}:=\mathrm{e}^{f(\cdot, X)} .
$$

Then, the $\mathbb{P}^{\prime}$-characteristics of the semimartingale $X$ are absolutely continuous and provided by $\left(b^{\prime}, c^{\prime}, F^{\prime}\right)$, where

$$
\begin{aligned}
b_{t}^{\prime} & =b_{t}+c_{t} \beta_{t}+\int_{\mathbb{R}^{d}}\left(Y_{t}(x)-1\right) h(x) F_{t}(\mathrm{~d} x) \\
c_{t}^{\prime} & =c_{t} \\
F_{t}^{\prime}(\mathrm{d} x) & =Y_{t}(x) F_{t}(\mathrm{~d} x),
\end{aligned}
$$

with $\beta_{t}=\mathrm{D} f\left(t, X_{t-}\right)$ and $Y_{t}(x)=\mathrm{e}^{f\left(t, X_{t-}+x\right)-f\left(t, X_{t-}\right)}$, for $t \in \mathbb{R}_{+}$and $x \in \mathbb{R}^{d}$.

Proof. The result follows directly from the previous lemma and Proposition 2.6 in Kallsen (2006).

\section{REFERENCES}

L. B. G. Andersen and V. V. Piterbarg. Interest Rate Modeling, 3 Vols. Atlantic Financial Press, 2010.

D. Belomestny, S. Mathew, and J. Schoenmakers. Multiple stochastic volatility extension of the LIBOR market model and its implementation. Monte Carlo Methods Appl., 15:285-310, 2009.

M. Bianchetti and M. Morini, editors. Interest Rate Modelling After the Financial Crisis. Risk Books, 2013.

A. Brace, D. Gạtarek, and M. Musiela. The market model of interest rate dynamics. Math. Finance, 7:127-155, 1997.

D. Brigo and F. Mercurio. Interest Rate Models: Theory and Practice. Springer, 2nd edition, 2006.

D. Criens, K. Glau, and Z. Grbac. Martingale property of exponential semimartingales: a note on explicit conditions and applications to asset price and LIBOR models. Preprint, arXiv: 1506.08127, 2015.

D. Duffie, D. Filipović, and W. Schachermayer. Affine processes and applications in finance. Ann. Appl. Probab., 13:984-1053, 2003.

E. Eberlein and W. Kluge. Exact pricing formulae for caps and swaptions in a Lévy term structure model. J. Comput. Finance, 9(2):99-125, 2006a.

E. Eberlein and W. Kluge. Valuation of floating range notes in Lévy term structure models. Math. Finance, 16:237-254, 2006b.

E. Eberlein and F. Özkan. The Lévy LIBOR model. Finance Stoch., 9:327-348, 2005. 
D. Filipović and A. Trolle. The term structure of interbank risk. J. Financ. Econom., 109:707-733, 2013.

P. Glasserman. Monte Carlo Methods in Financial Engineering. Springer, 2003.

T. Goll and J. Kallsen. Optimal portfolios for logarithmic utility. Stochastic Process. Appl., 89:31-48, 2000.

Z. Grbac and W. J. Runggaldier. Interest Rate Modeling: Post-Crisis Challenges and Approaches. SpringerBriefs in Quantitative Finance, Springer, 2015.

J. Jacod and A. N. Shiryaev. Limit Theorems for Stochastic Processes. Springer, 2nd edition, 2003.

F. Jamshidian. LIBOR and swap market models and measures. Finance Stoch., 1:293-330, 1997.

F. Jamshidian. LIBOR market model with semimartingales. Working Paper, NetAnalytic Ltd., 1999.

J. Kallsen. A didactic note on affine stochastic volatility models. In Yu. Kabanov, R. Lipster, and J. Stoyanov, editors, From Stochastic Calculus to Mathematical Finance: The Shiryaev Festschrift, pages 343-368. Springer, 2006.

J. Kallsen and A. N. Shiryaev. The cumulant process and Esscher's change of measure. Finance Stoch., 6:397-428, 2002a.

J. Kallsen and A. N. Shiryaev. Time change representation of stochastic integrals. Theory Probab. Appl., 46:522-528, 2002b.

M. Keller-Ressel, A. Papapantoleon, and J. Teichmann. The affine LIBOR models. Math. Finance, 23:627-658, 2013.

I. Klein, T. Schmidt, and J. Teichmann. No-arbitrage theory for bond markets. Preprint, 2015.

W. Kluge. Time-inhomogeneous Lévy processes in interest rate and credit risk models. PhD thesis, Univ. Freiburg, 2005.

W. Kluge and A. Papapantoleon. On the valuation of compositions in Lévy term structure models. Quant. Finance, 9:951-959, 2009.

M. Ladkau, J. Schoenmakers, and J. Zhang. Libor model with expiry-wise stochastic volatility and displacement. Int. J. Portfolio Analysis and Management, 1:224-249, 2013.

K. R. Miltersen, K. Sandmann, and D. Sondermann. Closed form solutions for term structure derivatives with log-normal interest rates. J. Finance, 52: 409-430, 1997.

M. Musiela and M. Rutkowski. Continuous-time term structure models: forward measure approach. Finance Stoch., 1:261-291, 1997.

M. Musiela and M. Rutkowski. Martingale Methods in Financial Modelling. Springer, 2nd edition, 2005.

A. Papapantoleon. Old and new approaches to LIBOR modeling. Stat. Neerlandica, 64:257-275, 2010.

A. Papapantoleon, J. Schoenmakers, and D. Skovmand. Efficient and accurate log-Lévy approximations to Lévy-driven LIBOR models. J. Comput. Finance, 15(4):3-44, 2012.

J. Schoenmakers. Robust LIBOR Modelling and Pricing of Derivative Products. Chapman \& Hall/CRC Press, 2005.

M. Wheatley. The Wheatley review of LIBOR. Technical report, HM Treasury, 2012. 
L. Wu and F. Zhang. LIBOR market model with stochastic volatility. $J$. Industr. Manag. Optim., 2:199-227, 2006.

Center for Mathematics, Technical University of Munich, Parkring 11, 85748 Garching B. MÜnChen, Germany

E-mail address: kathrin.glau@tum.de

Laboratoire de Probabilités et Modèles Aléatoires, Université Paris Diderot, 75205 PARIS CEDEX 13, France

E-mail address: grbac@math.univ-paris-diderot.fr

Institute of Mathematics, TU Berlin, Strasse des 17. Juni 136, 10623 Berlin, GERMANY

E-mail address: papapan@math.tu-berlin.de 